\title{
Mental and neurological health profile of Ecuador
}

\author{
Marcelo E. Cruz MD, ${ }^{1,2}$ Rachel Jenkins MD, ${ }^{1,3}$ Clare Townsend MD ${ }^{4}$ \\ and Donald Silberberg MD ${ }^{1,5}$
}

'Global Network for Research in Mental and Neurological Health, USA 2Panandean Corporation for Research and Development, Ecuador, and Ecuadorean Academy of Neurosciences ${ }^{3}$ Institute of Psychiatry, UK; ${ }^{4}$ University of Queensland, Australia; ${ }^{5}$ University of Pennsylvania, Philadelphia, USA

W e assessed the mental and neurological health (MNH) situation of Ecuador in 2006-8, using the Mental and Neurological Health Country Profile (MNHCP) (Gulbinat et al, 2004; Jenkins, 2004; Jenkins et al, 2004), an instrument which helps to develop evidence-based MNH policy and services (Townsend et al, 2004). An extensive review of the literature was undertaken and consultations and consensus meetings (Schilder et al, 2004) were conducted with key mental and neurological health stakeholders, including consumers, carers and clinicians from the government and non-government sectors.

\section{Context}

Ecuador, in the north-west of South America, has an area of $256370 \mathrm{~km}^{2}$ and a population of around 13400000 . The population distribution has become younger in recent decades, with $61.9 \%$ aged 15-64 years. Nearly two-thirds (63.4\%) of the population live in urban areas. The national fertility rate is 22.9 births per 1000 . Life expectancy is 78 years for women and 72 years for men. The major ethnic groups are Mestizo (65\%), Native Indigenous (25\%), White (7\%) and Black (3\%). Official languages are Spanish and Quechua. Most of the population (95\%) is Roman Catholic.

Other significant data are presented in Table 1.

Internal and external migration is significant. Over one million Ecuadorians emigrated to the USA and Europe following economic problems in 2000. The Colombian guerilla

Table 1 Select national data, 2006

\begin{tabular}{|c|c|}
\hline \multicolumn{2}{|l|}{ Measures } \\
\hline Gross national product & US\$44 billion \\
\hline National debt & US\$11 billion \\
\hline Economic aid & US\$22 billion \\
\hline Proportion of budget spent on health & $6 \%$ \\
\hline Proportion of budget spent on mental health & $0.00006 \%$ \\
\hline Annual income per capita & US\$3216 \\
\hline Proportion of population living below poverty line & $30 \%$ \\
\hline Proportion of homeless people & $31 \%$ \\
\hline $\begin{array}{l}\text { Proportion of population with access to safe } \\
\text { drinking water }\end{array}$ & $15 \%$ \\
\hline $\begin{array}{l}\text { Proportion of population with access to adequate } \\
\text { sanitation }\end{array}$ & $43 \%$ \\
\hline Unemployment rate & $8.8 \%$ \\
\hline Inflation rate & $3.1 \%$ \\
\hline Child immunisation coverage & $93 \%$ \\
\hline Prison population & $\begin{array}{l}14400 \text { male, } \\
1600 \text { female }\end{array}$ \\
\hline
\end{tabular}

Source: Inec, Indicadores Basicos de Salud (2006). movement has forced the displacement of half a million Colombians into Ecuador and rural migration continues to increase the size of the slum areas in major cities.

Positive mental health and well-being is understood as emotional health; mental disorder and mental illness as loss of reason; neurological illness is described in terms of the associated disability; and personality disorder is understood as antisocial or delinquent behaviour. Discrimination against people with mental or neurological illnesses exists owing to cultural influences and ignorance. Mental and neurological disorders are considered physical and cultural ailments, and approximately $30 \%$ of the population (particularly those in the rural sector) regard mental and neurological conditions as punishments of nature.

Women primarily bear the burden of caring for people with mental and neurological disorders. There are significant levels of stigma and discrimination shown to people with $\mathrm{MNH}$ problems and to other vulnerable groups, including those who experience physical or intellectual disability, poverty, disease, the young and the aged. There is no recognition of the rights of people with disabilities, resulting in government failure to address the physical and $\mathrm{MNH}$ needs of these groups, until a start was made by the 2006 Organic Health Law.

Violence against women, associated with male jealousy and macho attitudes, is common. Alcohol misuse is high and commonly associated with unemployment, lack of close family, marital problems and debt. Drug misuse among children and young people is rising. This is frequently associated with parental migration, as it often causes young people to come under the care of grandparents. Homicide rates have tripled in the last 19 years, going from 6.5 per 100000 people in 1985 to 19 per 100000 in 2004. Drug- and alcohol-related criminal violence resulted in 2315 deaths in 2004.

\section{Social policy, health policy, legislation and human rights}

Ecuador experienced social spending restrictions, economic reforms and economic restructuring in the 1980s, followed by the introduction of welfare legislation in 1992 and compulsory education in 2000. However, school non-attendance remains high. Alcohol is available only to those above 18 years of age; public tobacco advertising is restricted; and tobacco and alcohol are taxed. There is regulation of drink-driving and firearms.

Free maternity services are provided and there are childhood immunisation programmes. There are, however, no 
programmes aimed at: reducing substance misuse, tobacco or alcohol consumption; detecting and preventing high blood pressure; or promoting life skills in schools.

There is no national MNH policy in Ecuador. The Ministry of Public Health is the main body responsible for the regulation of MNH care. The 2006 Organic Health Law legislates for human rights in the field of $\mathrm{MNH}$, and regulates the way in which involuntary admission is decided by family members, physicians, police officers and the justice system. The law guarantees patients access to their case-notes, but there is no tradition of enforcing constitutional rights.

\section{Estimates of need}

Small-scale neuroepidemiological surveys were carried out in Ecuador in the 1980s, using a World Health Organization protocol (Cruz et al, 1985). Epilepsy prevalence figures (10-17/1000) are generally higher than those found in industrialised countries. Migraine headache has been found to affect 68 people per 1000 (Cruz et al, 1995). The prevalence of established cerebrovascular disease was 3.5 per 1000 and of peripheral neuropathies 15 per 1000 . There are no community-level epidemiological data on mental disorders.

\section{Financial and human resources}

The main obstacle to the purchasing and delivery of specialist care in Ecuador is lack of financial resources. Central government spending accounts for only a small proportion of national expenditure for mental and neurological illnesses. In 2008, 6\% of the national budget (US $\$ 700$ million) was allocated to general health. Of this, US\$3200000 was allocated to $\mathrm{MNH}$. MNH services are also financed through social security insurance, private insurance, fee for service, community financing, charity and donor funds (loans and grants). The cost of medicines is high, public health services are limited and private sector services are expensive. The approximate financial burden experienced by a family when a member is mentally or neurologically ill is approximately US $\$ 500$ per month.

Ecuador has 4658 primary care doctors, 338 psychiatrists (or 2.3 per 100000), 333 neurologists, 22 community psychiatric nurses and 1 psychiatric social worker.

Medical students receive 36 hours of training in psychiatry and 36 hours in neurology, spread over the 18 weeks of the internal medicine module. Psychiatric postgraduate training has been in place for the past 25 years, sponsored by the Central University of Ecuador, in Quito. It is a 3-year course, with eight students per year. A similar programme has been established recently by the University of Cuenca. There are no training programmes in psychiatric nursing.

\section{Services}

Initial treatment is generally in the form of home-made medicines, products from local pharmacies or friends' advice. There are 721 primary care centres (169 in the state sector, 12 in the non-government sector and 540 private facilities). Specialist care is also available.

There are 1635 psychiatric beds in state-funded general and teaching hospitals. No information is available on the non-governmental and private provision of psychiatric beds.

It is a legal provision that psychotropic substances are available only through prescription. In Ecuador the financial expenditure on antidepressants and anticonvulsants alone is about US\$120 million per year.
The 2006 Organic Health Law integrates traditional medicine within the national health system. Traditional healers include 'cleansers' (who clean the body of bad spirits), midwives, healers, shamans and witches. Other alternative/complementary medicine includes homeopathy, acupuncture and bioenergetics.

There are no programmes for $\mathrm{MNH}$ promotion or plans for training workers in this field. There are no policies for reducing mortality from physical illnesses in people with a mental or neurological illness, or initiatives to address suicide reduction or epilepsy and stroke prevention. There are no programmes for addressing the needs of children, older people or disadvantaged minorities, and initiatives are limited in areas such as incest, child abuse, domestic violence, trauma and rape.

The prison population totals 16000 , of whom $80 \%$ fulfil ICD-10 criteria for mental disorders and 10\% deserve specialist attention. Medical services and therapeutic communities exist within prisons. Prisoners have access to primary care and psychiatric and psychological evaluation and in an emergency may be referred to specialist psychiatric services. There are protocols within the prisons for prevention of violence and crisis intervention. Two psychiatrists and 60 psychologists work in the prisons.

\section{Discussion}

Key problems for $\mathrm{MNH}$ in Ecuador include limited resources and services. The main challenge is to establish a national $\mathrm{MNH}$ policy with widespread ownership by the key stakeholders. MNH policy needs to address stigma and discrimination, health promotion and illness prevention, and improved supply of and access to clinical and non-clinical services. MNH needs to be integrated into primary care, with supervision and support from specialist services which are decentralised to all districts, more specialised multidisciplinary personnel, and better access to low-cost medicines. Funding strategies are needed to address resource generation, allocation and audit. Access to care is restricted owing to scarce financial resources, cultural and religious beliefs, a weak primary care system, long distances from the main hospitals, concentration of specialist services in the main cities, discrimination and a lack of inter-sectoral liaison at local and national levels, including within public policy. Of particular concern is the total lack of research in this area or plans for training separate cadres of specialist care staff.

\section{Conclusion}

Ecuador has significant $\mathrm{MNH}$ needs, aggravated by rising levels of emigration, and high levels of alcohol misuse and violence. Mental health services are mainly delivered by specialists, concentrated in the cities. There is a pressing need for decentralisation of services, for systematic support to primary care, and for inter-sectoral liaison in order to enhance access to mental health promotion, prevention and treatment.

\section{References}

Cruz, M. E., Schoenberg, B. S., Ruales, J., et al (1985) Pilot study to detect neurologic disease among a population with high prevalence of endemic goiter. Neuroepidemiology, 4, 108-116. 
Cruz, M. E., Cruz, I., Preux, P-M., et al (1995) Headache and neurocysticercosis in Ecuador, South America. Headache, 35, 93-97.

Gulbinat, W., Manderscheid, R., Baingana, F., et al (2004) The International Consortium on Mental Health Policy and Services: objectives, design and project implementation. International Review of Psychiatry, 16, 5-17.

Jenkins, R. (ed.) (2004) International project on mental health policy and services. Phase 1: Instruments and country profiles. International Review of Psychiatry, 16(1-2).

Jenkins, R., Gulbinat, W., Manderscheid, R., et al (2004) The Mental Health Country Profile: background, design and use of a systematic method of appraisal. The International Consortium on Mental Health
Policy and Services: objectives, design and project implementation. International Review of Psychiatry, 16, 31-47.

Schilder, K., Tomov, T., Mladenova, M., et al (2004) The appropriate and use of focus group methodology across international mental health communities. The International Consortium on Mental Health Policy and Services: objectives, design and project implementation. International Review of Psychiatry, 16, 24-30.

Townsend, C., Whiteford, H., Baingana, F., et al (2004) The Mental Health Policy Template: domains and elements for mental health policy formulation. The International Consortium on Mental Health Policy and Services: objectives, design and project implementation. International Review of Psychiatry, 16, 18-23.

\title{
First-episode psychosis: factors associated with delayed access to care in a rural Egyptian setting
}

\author{
Mamdouh El-Adl, ${ }^{1}$ Mohammed El-Mahdy ${ }^{2}$ and Musheera Anis ${ }^{3}$
}

${ }^{1}$ Consultant Psychiatrist, Northamptonshire Healthcare NHS Trust, Northampton, UK, email Mamdouh.eladl@nht.northants.nhs.uk ${ }^{2}$ Assistant Professor of Psychiatry, Al-Azhar University, Dhumyatt, Egypt; ${ }^{3}$ Psychiatric Specialist, Mansoura General Hospital, Egypt

0 ver the past few decades there has been a growing interest in first-episode psychosis (FEP), help-seeking behaviour and pathways to care. Treating psychotic disorders in their earliest stages has become a key focus for research and clinical care (Yung \& McGorry, 2007). FEP studies show that the average time between onset of symptoms and first effective treatment is often 1 year or more (McGlashan, 1987). This long duration of untreated psychosis (DUP) is undesirable. Early treatment helps minimise the risk of the serious consequences of untreated psychosis, in terms of changes in mental state and behaviour (Larsen et al, 1998; Wyatt et al, 1998) and can reduce suffering (Ho et al, 2003). Some early results suggested that an 'early intervention in psychosis' (EIP) service is more cost-effective than generic services (Mihalopoulos et al, 1999).

Most FEP studies have been conducted in high-income countries. The study reported here addressed the question of delayed access to care for an FEP in a rural Egyptian setting.

\section{Method}

The study was approved by Al-Azhar University research and ethics committee. Consent was taken from patients and their families before they took part in the study.

All patients with an FEP (meeting ICD-10 criteria) presenting consecutively to the psychiatric department at Mansoura General Hospital (MGH) between January and June 2004 were interviewed. Patients and relevant others (family or friends) were interviewed either separately or together at first contact or after improvement of the acute stage.

The interview protocol consisted of a semi-structured, userfriendly interview which included seven main sections that required only the ticking of various options. In every section there was an additional space under '(Other)' to allow more flexibility and to allow the inclusion of any additional information. The seven sections were: patient's details; mode of referral; referred to out-patient or in-patient department; DUP; family history of mental illness; diagnosis and treatment; and causes for delayed contact with the psychiatric service.

The study was carried out in Mansoura city, the capital of Eldakahlia province; it is surrounded by a large rural area which includes about 15 towns. The population of Eldakahlia is about 8 million, some 2 million of whom live in Mansoura. In Egypt, primary care is still underdeveloped. Therefore, access to secondary care is mainly not through primary care. Individuals who need specialist care are likely to present to the specialist clinic (state or private) based on their own perception of the nature of the problem or as advised by family or a friend. Two main medical centres cover healthcare in Eldakahlia: Mansoura University Hospital (MUH) and $\mathrm{MGH}$. Each has a psychiatric department. The smaller hospitals (called central hospitals, of which there is one in each town or big village) have no psychiatric department. Therefore, the mental health service in Egypt is essentially hospital based, and there is no well developed community mental health service.

The psychiatric department at $\mathrm{MGH}$ has a catchment of about 1 million. It contains an eight-bed ward and an out-patient clinic attended by about 100 patients per day. The medical staff includes two senior house officers, eight specialists and two consultant psychiatrists, and the nursing and non-medical staff comprises eight psychiatric nurses, a psychologist and one social worker.

\section{Results}

Of the 43 patients referred to the study, 40 agreed to take part ( 2 refused admission and decided to seek treatment privately and 1 refused to take part in the study). Out of the 40 patients, 22 needed admission (6 under section and 16 informal) and 18 were treated as out-patients. 